Plant Syst. Evol. 233: 15-28 (2002)

DOI $10.1007 / \mathrm{s} 00606-002-0213-1$

Plant Systematics and Evolution

Printed in Austria

\title{
Evolutionary history of the polyploid complex of Cardamine amara (Brassicaceae): isozyme evidence
}

\author{
K. Marhold ${ }^{1,2}$, M. Huthmann ${ }^{3,4}$ and H. Hurka ${ }^{3}$ \\ ${ }^{1}$ Institute of Botany, Slovak Academy of Sciences, Bratislava, Slovakia \\ ${ }^{2}$ Department of Botany, Charles University, Prague, Czech Republic \\ ${ }^{3}$ Department of Systematic Botany, University of Osnabrück, Germany \\ ${ }^{4}$ Present address: Institute of Botany, University of Agricultural Sciences, Vienna, Austria
}

Received June 22, 2001; accepted May 17, 2002

Published online: September 13, 2002

(C) Springer-Verlag 2002

\begin{abstract}
In samples from 56 populations of Cardamine amara, representing four diploid subspecies (subspp. amara, opicii, balcanica, and pyrenaea) and two tetraploid subspecies (subspp. austriaca and olotensis) from different parts of the European distribution area, four enzyme systems with 23 alleles were studied. These data, together with previous morphological and karyological data, suggest that the distribution and variation pattern within the species was strongly influenced by the last glacial period and postglacial migrations. Cardamine amara subsp. pyrenaea is monomorphic for a unique allele, and subsp. balcanica has a unique allele, too which, however, is not fixed in all populations of the taxon. Both taxa seem to be relic ones, although otherwise subsp. balcanica in respect of allelic spectrum much resembles subsp. amara. The other two diploid subspecies, subsp. amara and subsp. opicii, are not characterised by presence of unique alleles but differ in allele frequencies. The two tetraploid subspecies have different evolutionary histories. C. amara subsp. austriaca seems to be an autopolyploid derivative of subsp. amara which colonised open space offered by retreating glaciers in the Eastern Alps. C. amara subsp. olotensis from the Iberian Peninsula represents most probably a polyploid of preglacial time.
\end{abstract}

Key words: Cardamine amara, Large Bitter-cress, isozymes, polyploidy, glaciation, relic taxa.

\section{Introduction}

Cardamine L. comprises several polyploid complexes in its European distribution area, e.g. the $C$. pratensis group, $C$. amara L. and the $C$. raphanifolia group. Until now detailed attention has been paid mainly to the C. pratensis complex, which consists of several diploid taxa and higher polyploids up to dodecaploid level, including dysploids and aneuploids (e.g. Lövkvist 1956; UrbanskaWorytkiewicz and Landolt 1974; Marhold 1994a,b, 1996; Marhold and Ančev 1999; Franzke and Hurka 2000).

Recent studies of populations of $C$. amara in various parts of Europe revealed an interesting pattern of karyological and morphological variation classified at the subspecific level (Lihová et al. 2000; Marhold 1992, 1999; Marhold et al. 1996). Four diploid subspecies are currently recognised within $C$. amara: subsp. amara, widespread in most of Europe 
extending to Asia, except some European mountain ranges where it is replaced by other subspecies; subsp. opicii (J. Presl et C. Presl) Celak. occurring at the higher altitudes of the Sudety Mts. and Carpathians (Marhold 1992) (for the distribution of all subspecies in studied part of Europe see Fig. 1); subsp. balcanica Marhold, Ančev et Kit Tan, occurring in the mountains of SW Bulgaria and NE Greece (Marhold et al. 1996); and subsp. pyrenaea Sennen, a recently "rediscovered" taxon of the Eastern Pyrenees (Lihová et al. 2000). There are two tetraploid subspecies in $C$. amara. They occupy different restricted areas: subsp. austriaca Marhold occurs in the Eastern Alps and neighbouring areas (Marhold 1999), while subsp. olotensis occupies a small area in Catalonia (Lihová et al. 2000). The results of random amplified polymorphic DNA (RAPD) analyses of all six subspecies of Cardamine amara (Lihová et al. 2000) based on a restricted amount of material supported the abovementioned taxonomic treatment except that diploid subsp. amara and tetraploid subsp. austriaca were not differentiated from each other. This can be explained by the possible autotetraploid origin of subsp. austriaca from subsp. amara. C. amara subsp. pyrenaea, subsp. opicii, subsp. balcanica, and especially subsp. olotensis formed well-defined groups in

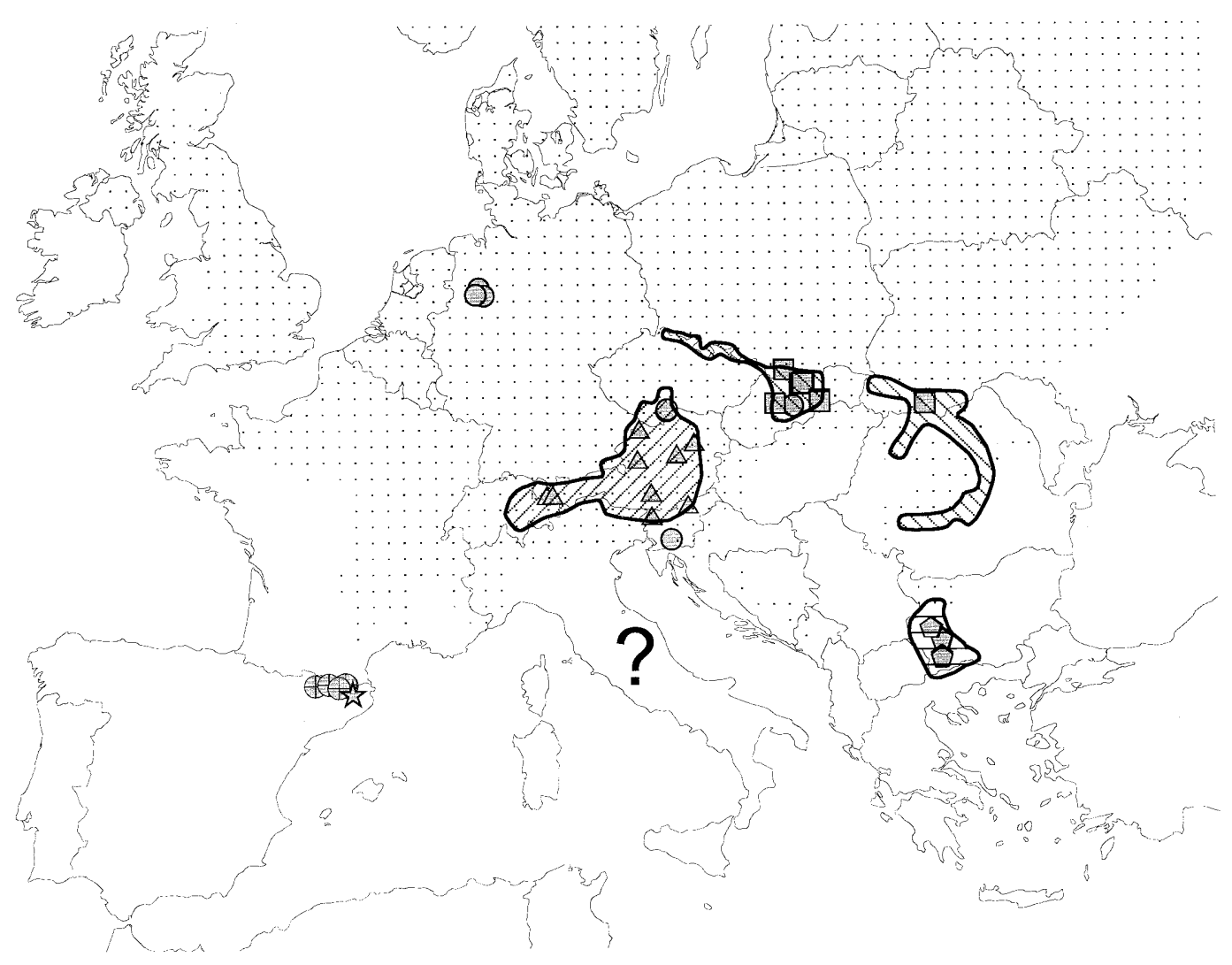

Fig. 1. Map of distribution of sample sites, and distribution areas, of Cardamine amara subsp. amara (circle $\bigcirc$, $\ldots$, representing the approximate distribution area, which further extends to the east to Asia, in some parts of the Balkan Peninsula the detailed distribution is still poorly known), C. amara subsp. opicii (square $\square, \mathbf{\Delta}$ ), C. amara subsp. austriaca (triangle $\triangle$, $\mathbf{Z 7}$, only marginally overlapping with the area of subsp. amara), C. amara subsp. balcanica (pentagon $\triangle, \Longrightarrow$ ), C. amara subsp. olotensis ( $\operatorname{star} \succsim$ ), and C. amara subsp. pyrenaea (crossed circle $\oplus$ ) (distribution areas of the latter two subspecies coincide with distribution of sample sites). The taxonomic status of the central Italian populations is uncertain and it is being studied now 
neighbour joining-distance analysis as well as in principal coordinate analysis of the RAPD data.

The distribution area of $C$. amara subsp. austriaca is clearly influenced by the migrations and evolutionary processes connected with Pleistocene glaciation. Its occurrence is centred in the area covered by the glacier during the last glaciation and for most of this area it represents the only representative of this species; its establishment was very likely connected with the quick occupation of these areas after retreat of the glacier. The distribution of taxa influenced by glaciation and postglacial migrations is documented in recent literature, especially in connection with the use of various kinds of molecular methods (e.g. Taberlet et al. 1998; Hewitt 1999, 2000).

Franzke and Hurka (2000) analysed isozymes, internal transcribed spacer (ITS), noncoding chloroplast DNA, and RAPDs to elucidate phylogenetic relationships and historical biogeography of the Cardamine pratensis complex, closely related to the group studied in the present paper.

The aim of the present paper is to analyse isozyme variation in Cardamine amara for phylogenetic and historical biogeographic signals.

\section{Material and methods}

Plant material. Living plants of Cardamine amara L. comprising all the currently recognised six subspecies were collected in the field and transferred to the greenhouse of the University of Osnabrück, Germany, where they were grown under uniform conditions (for list of localities see Table 1 and for their distribution Fig. 1). Young leaves were harvested and stored at $-80{ }^{\circ} \mathrm{C}$. Samples from 56 populations or sub-populations (altogether 351 plants, see Table 1) of C. amara were studied. Voucher specimens are deposited in the herbarium SAV.

Chromosome counts. Chromosome numbers of the studied populations are presented in Table 1. In most cases they are previously published counts (Lihová et al. 2000; Marhold 1994a, 1999; Marhold et al. 1996). In a few cases they represent new data by Marion Huthmann counted by the procedure described in Koch et al. (1996: 588), on at least ten plants from each locality. Voucher specimens for these counts are deposited in WHB.

Isozyme analysis. The following enzyme systems were assayed: aspartate aminotransferase (AAT, EC 2.6.1.1); glutamate dehydrogenase (GDH, EC 1.4.1.2-4); leucine aminopeptidase (LAP, EC 3.4.11.1); phosphoglucomutase (PGM, EC 2.7.5.1). Extracts were prepared from $0.5 \mathrm{~g}$ frozen leaves of single plants in $1 \mathrm{ml}$ ice-cold extraction buffer $(0.165 \mathrm{M}$ Tris- $\mathrm{HCl}, \mathrm{pH} 8.0$, and $0.107 \mathrm{M}$ glycine), and $200 \mathrm{mg}$ Amberlite IRA-401 (Serva, Heidelberg) were added. The extract was filtered through four layers of mull and centrifuged at $4{ }^{\circ} \mathrm{C}$ for $30 \mathrm{~min}$ at $20,000 \mathrm{rpm}$. The supernatant was stored at $-80{ }^{\circ} \mathrm{C}$. Electrophoresis was performed in a continuous system on $5.5 \%(\mathrm{w} / \mathrm{v}$, GDH) and 7.5\% (w/v, AAT, LAP, PGM) polyacrylamide gel using different buffer systems and staining procedures (see Franzke and Hurka (2000) for AAT, GDH, and LAP; Mummenhoff and Hurka (1995) for PGM). The allozymes were characterised by their $R_{f}$ values relative to an internal standard with a migration distance of 100 (=allele 1 of the respective locus). The term allozyme is used in the present paper also for tetraploids for simplicity, although no genetic analysis of inheritance patterns has been carried out.

Data analyses. For the samples from the diploid populations (subsp. amara, subsp. opicii, subsp. balcanica, and subsp. pyrenaea), allele frequencies within populations were determined, and genetic (chord) distances were calculated according to Cavalli-Sforza and Edwards (1967) (for the arguments in favor of this distance measure compared to distance according to Nei $(1972,1978)$ see Koch and Hurka (1999) and references therein). For the tetraploid taxa (subsp. austriaca and subsp. olotensis) frequency data presented in Results section reflect presence/absence of alleles in individuals rather than actual allelic frequencies as it was hard to evaluate the number of alleles in particular polyploid individuals based on intensity of bands. Therefore only the presence and absence of alleles was used for numeric evaluation of tetraploid populations. Matrix of frequencies for diploid populations, and a matrix of presence/ absence data for diploid and tetraploid populations were subjected to principal component analysis. 
K. Marhold et al.: Isozyme diversity in the Large Bitter-cress

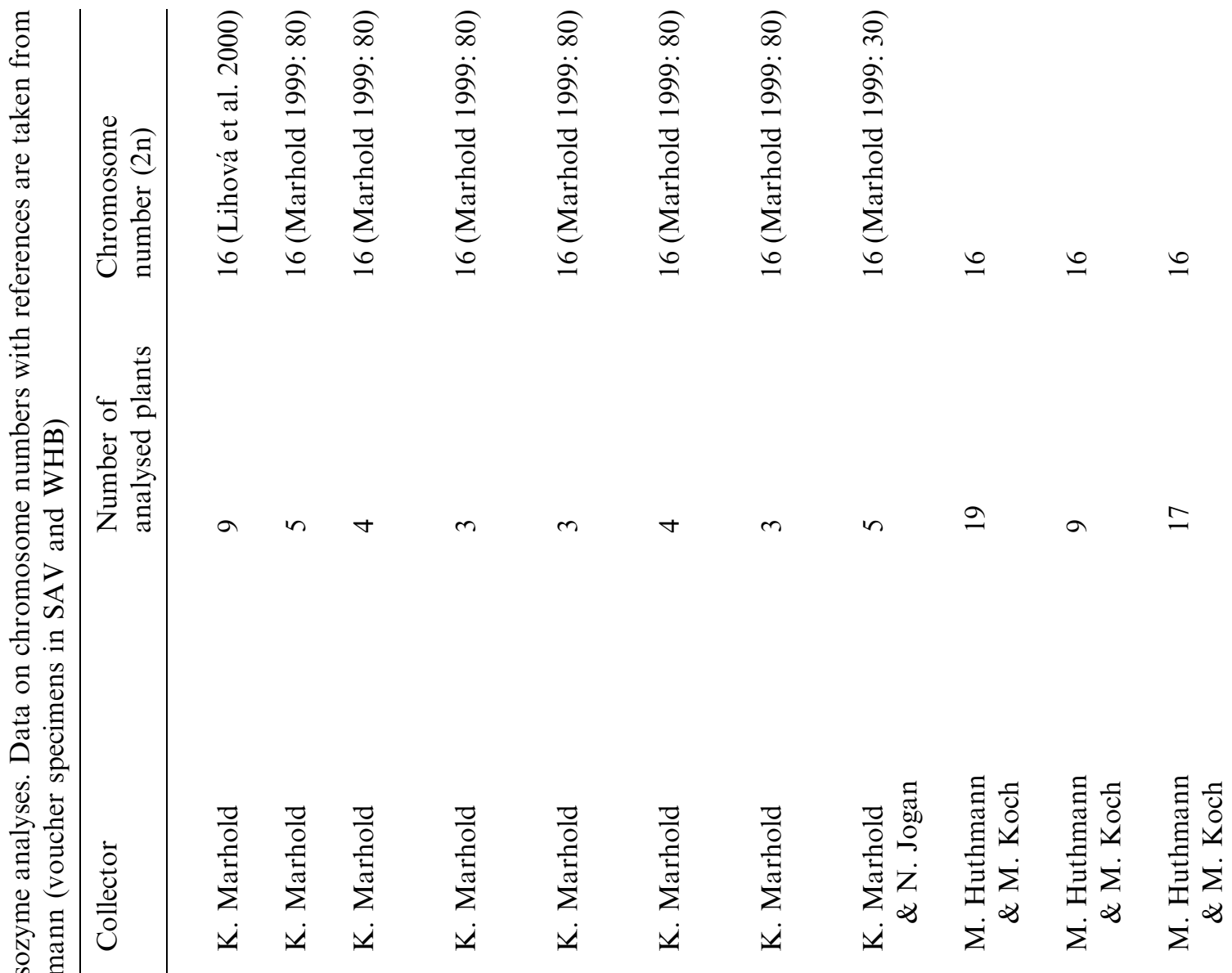

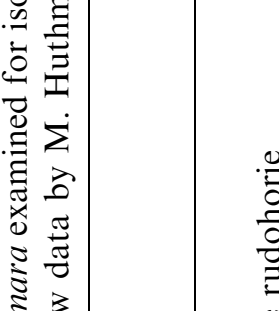

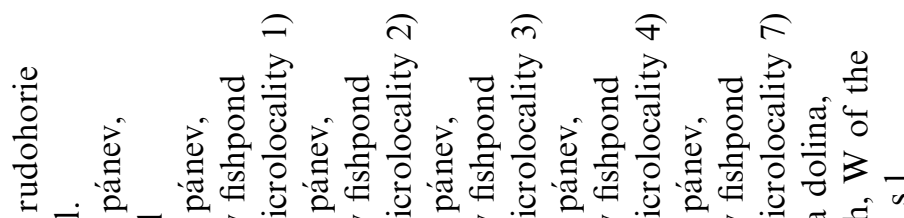

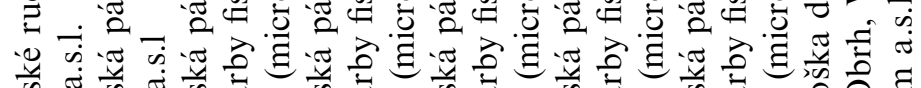

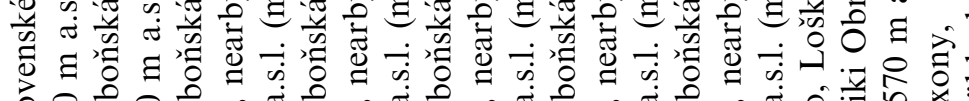

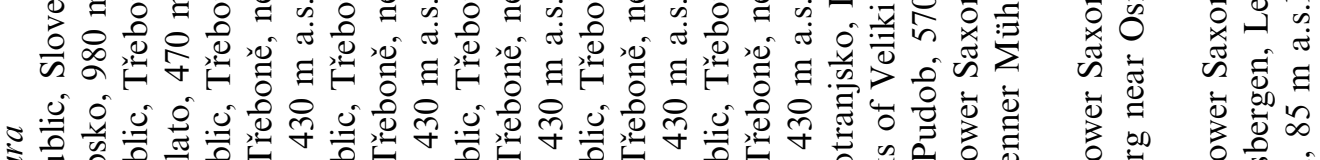

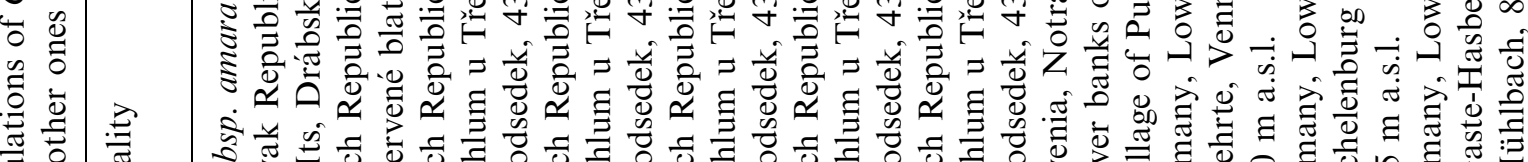

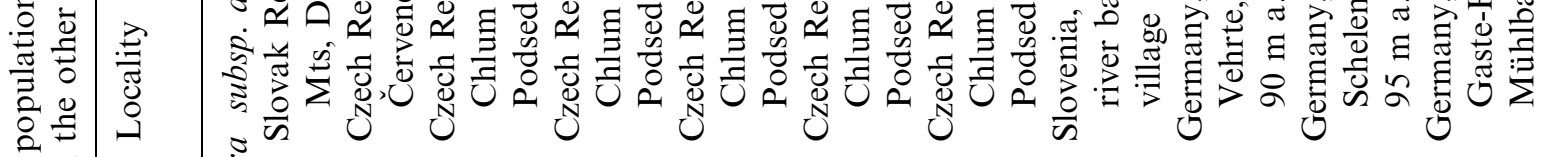

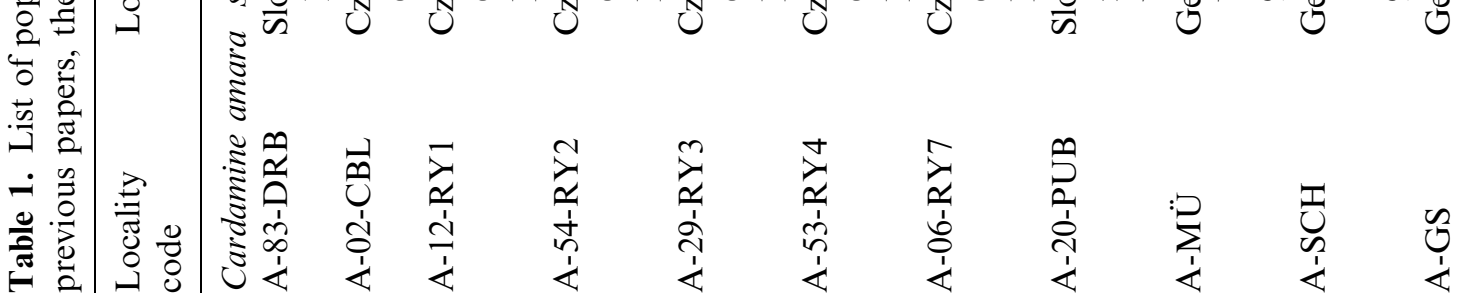


K. Marhold et al.: Isozyme diversity in the Large Bitter-cress

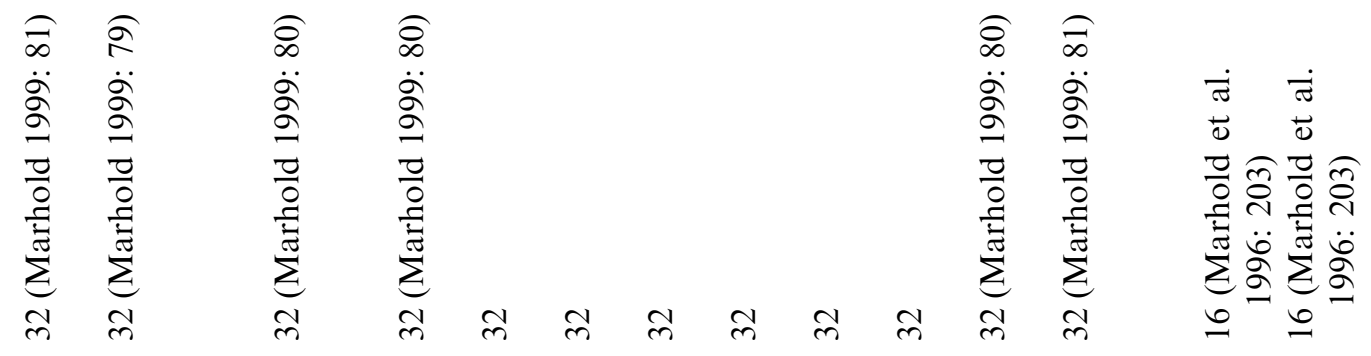

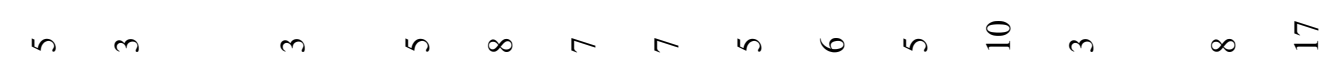

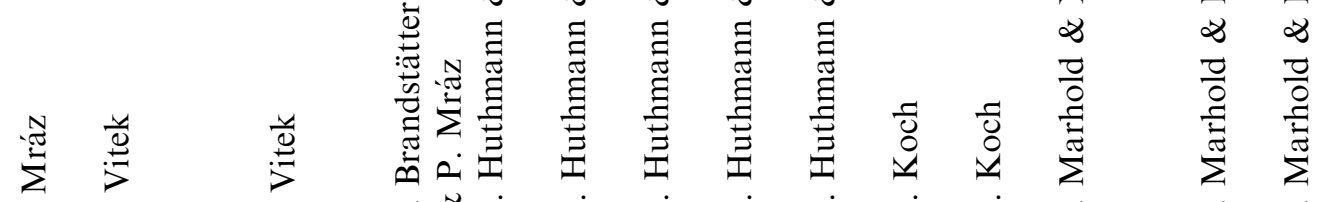

म ए

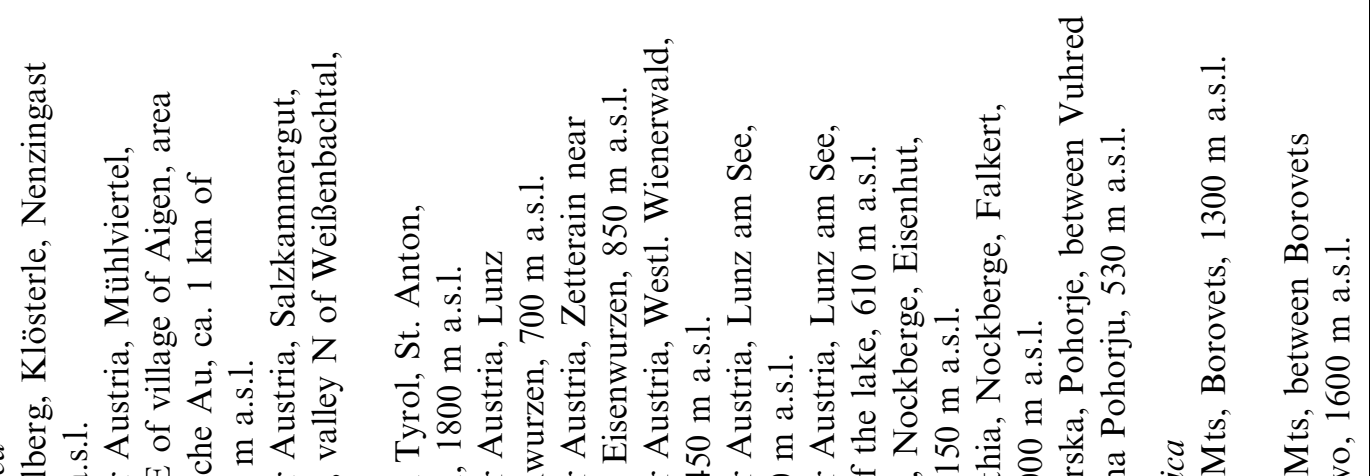

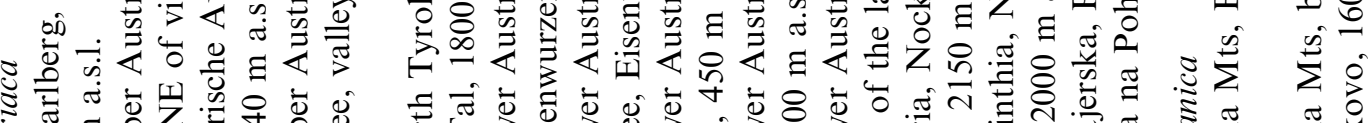

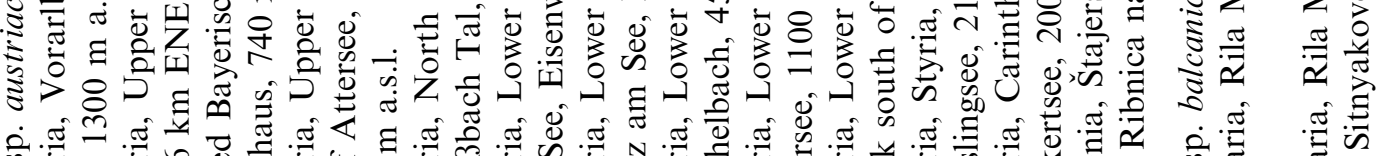

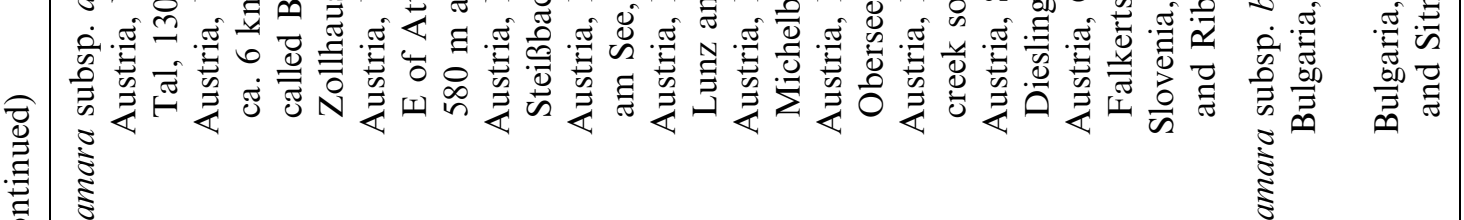
离

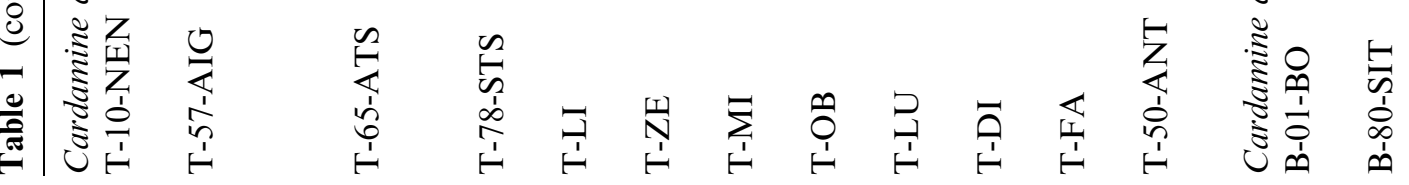


K. Marhold et al.: Isozyme diversity in the Large Bitter-cress

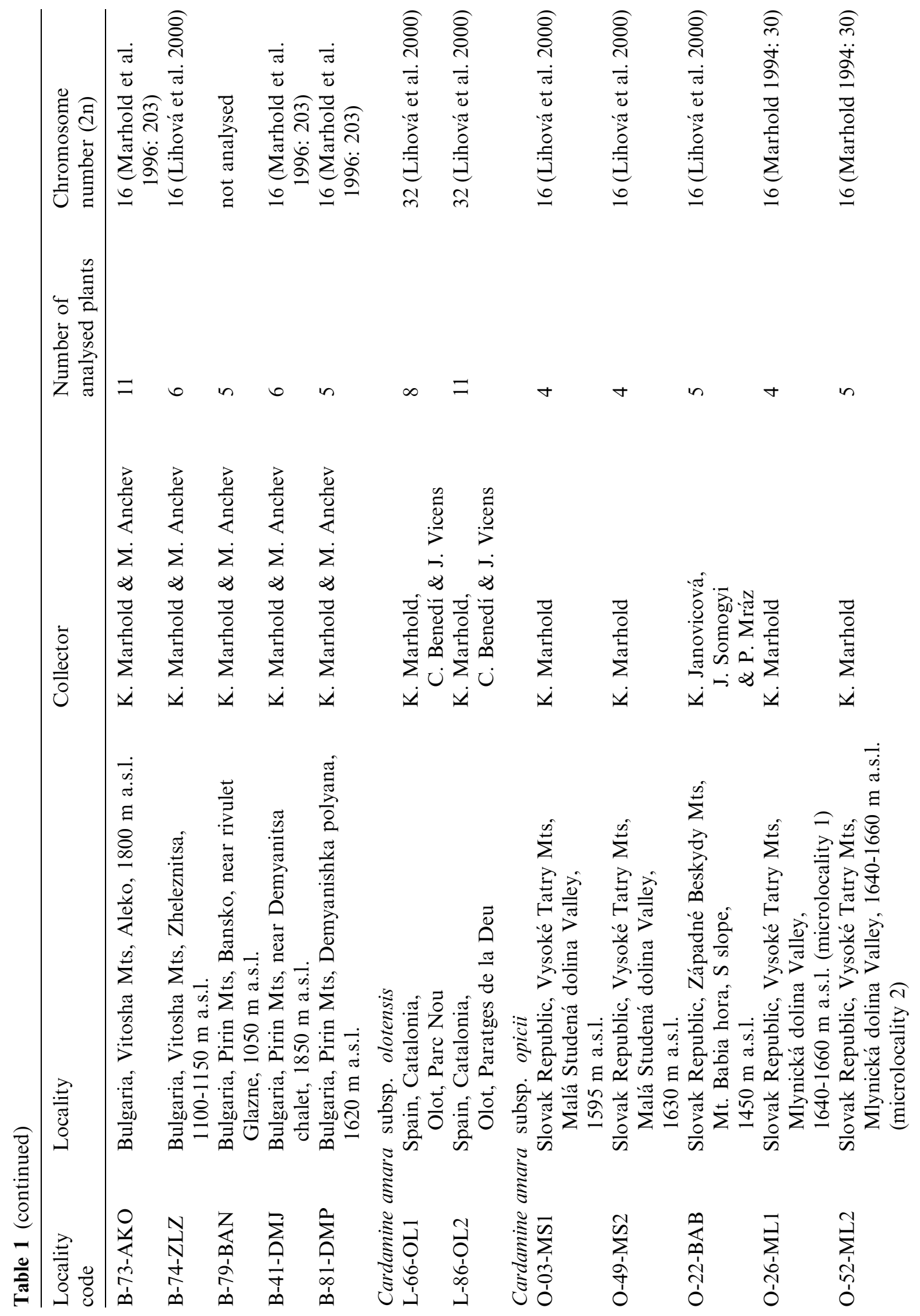


K. Marhold et al.: Isozyme diversity in the Large Bitter-cress

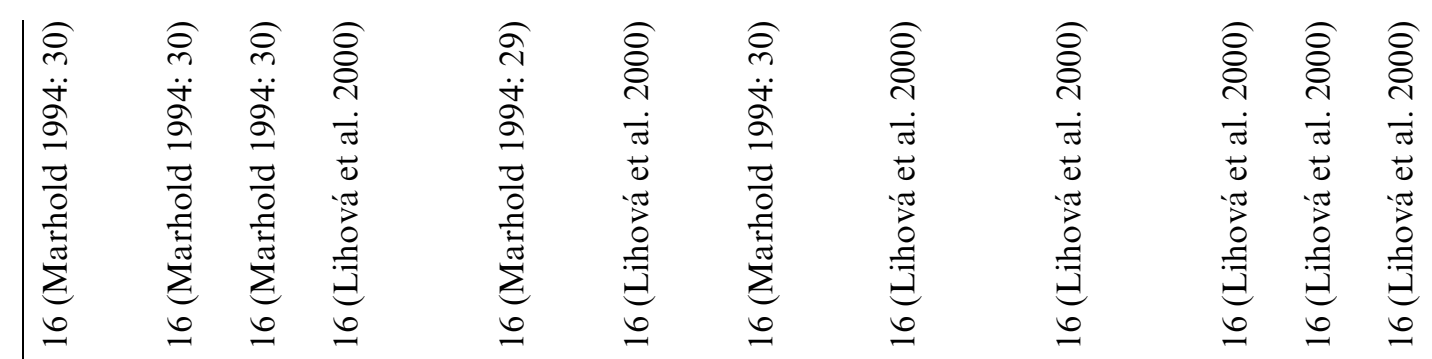

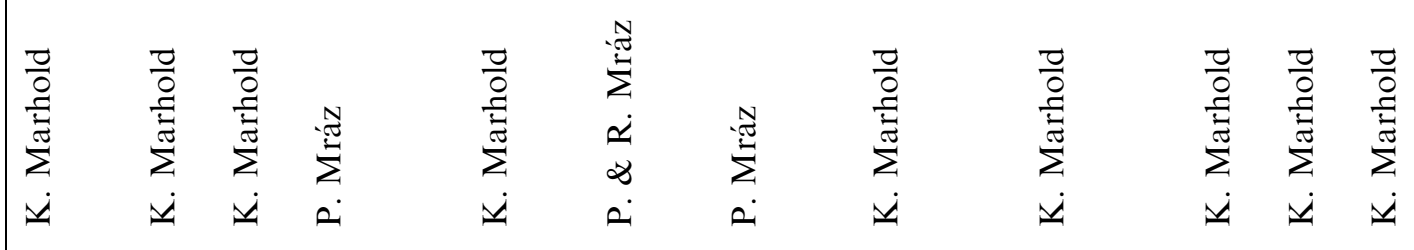

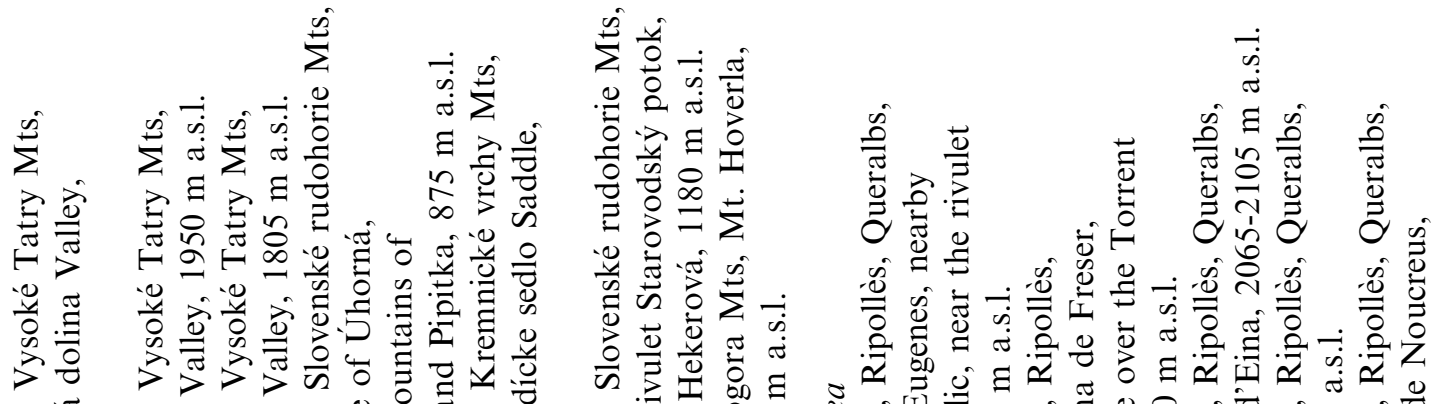

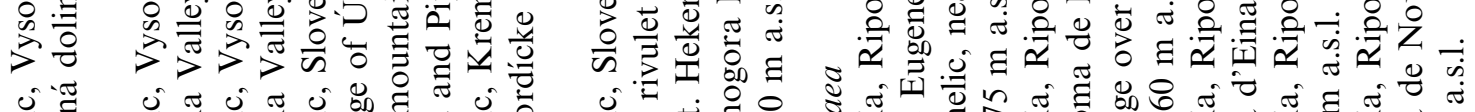

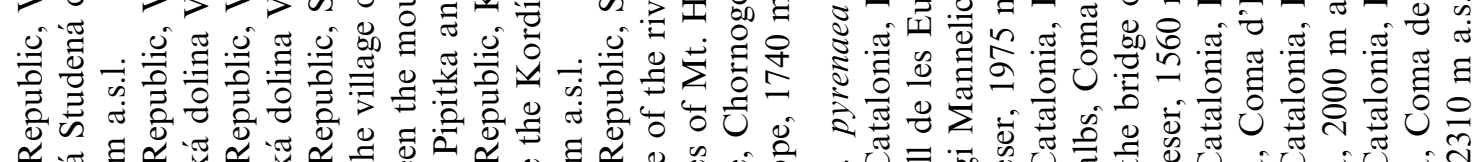

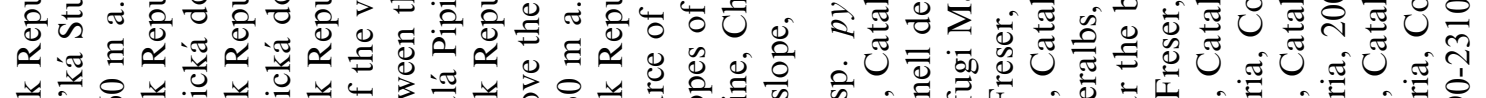

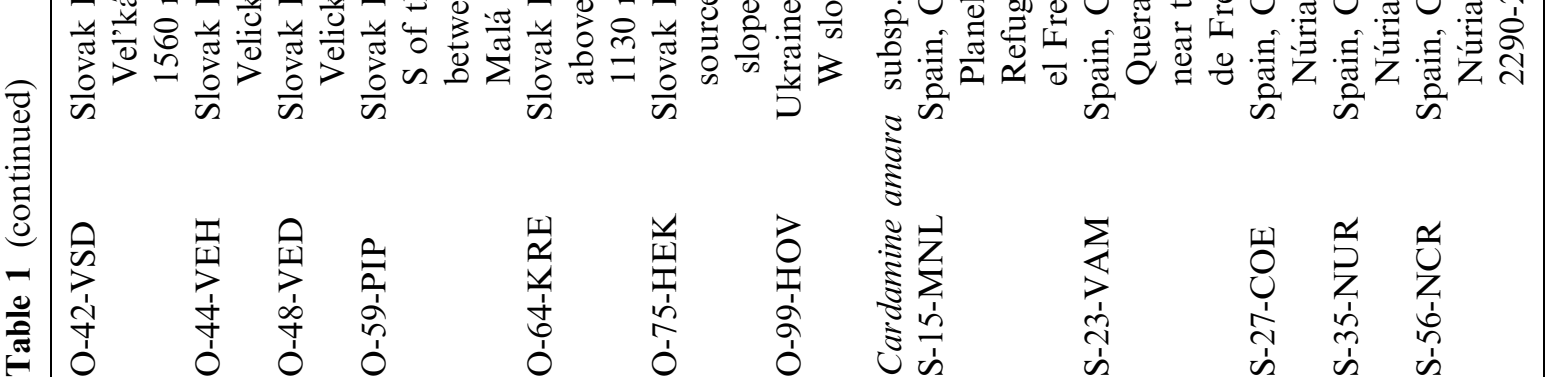


K. Marhold et al.: Isozyme diversity in the Large Bitter-cress

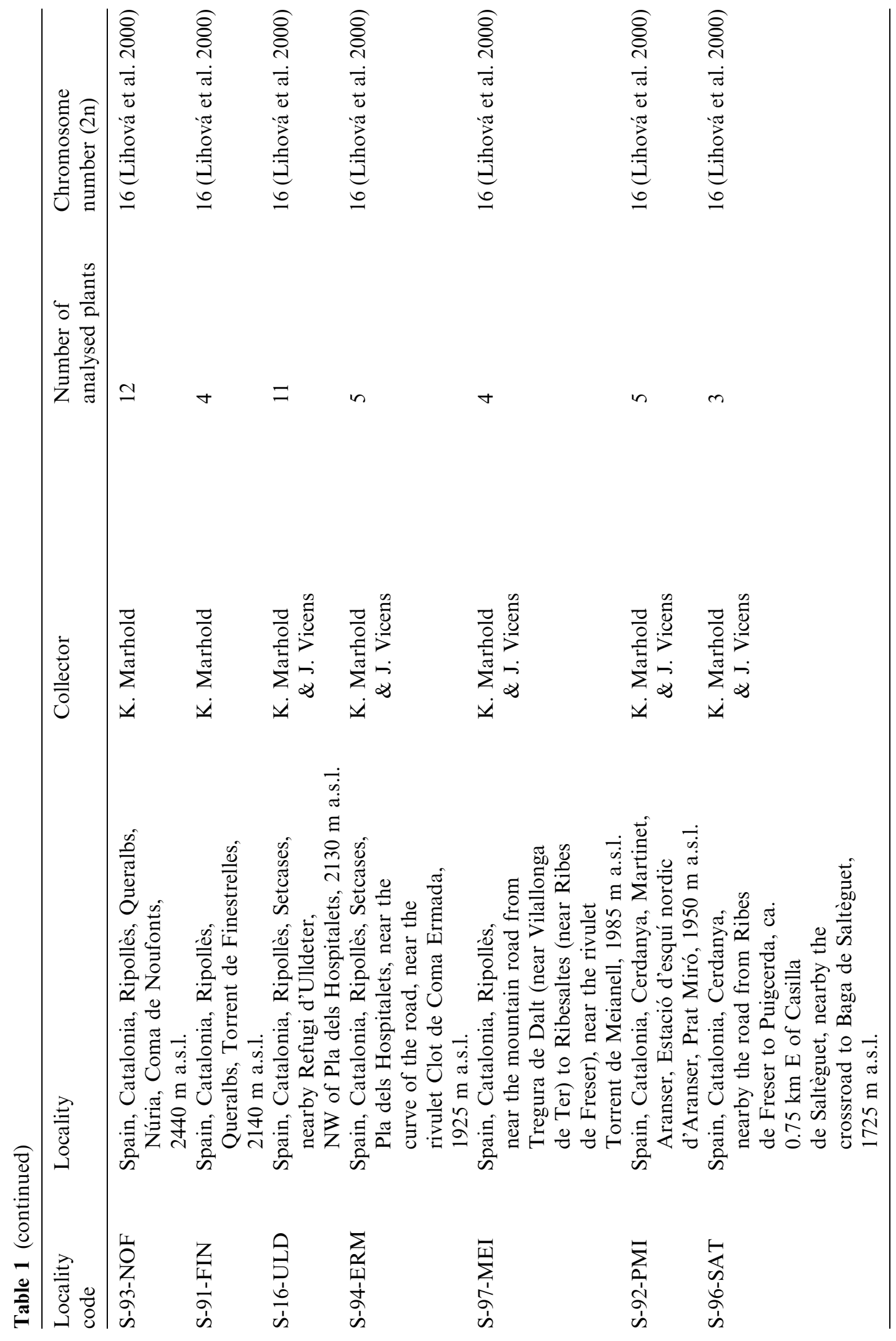


The matrix of presence/absence data for diploids and tetraploids was also subjected to the nonparametric k-nearest neighbours classificatory discriminant analysis (Klecka 1980) using crossvalidation and currently recognised subspecies as groups. This method develops classificatory criterion based on $n-1$ cases (out of total $n$ cases included in the analysis) and this criterion is in turn applied to the case left out. The whole procedure is repeated $\mathrm{n}$ times, and estimates extent of separation of the classified groups. Based on the distance matrix for the diploid population samples, a phenogram using UPGMA (unweighted pairgroup arithmetic average clustering, average linkage) algorithm and an ordination diagram using principal coordinate analysis (PCO) were generated. The computations were performed with the SAS (SAS Institute 1990a,b), BIOSYS-1 version 1.7 (Swofford and Selander 1989), and SYN-TAX 2000 (Podani 2001) statistical program packages.

\section{Results}

Isozyme analysis. The distribution of alleles at the four enzyme systems analysed (AAT, GDH, LAP, PGM) among all taxa (diploid and tetraploid) of Cardamine amara are shown in Table 2 as presence/absence data, and in Table 3 as allele frequencies.

Two loci with 6 alleles were analysed for AAT (Aat1 and Aat2). Allele Aat2-7 was unique for subsp. pyrenaea and fixed in all accessions studied. Allele Aat2-4 was found exclusively in subsp. balcanica but detected only in three of the seven surveyed populations. Two loci with 6 alleles coded for PGM (Pgm1 and Pgm2). Allele Pgm2-1 was discovered only in subsp. olotensis. It was fixed in that subspecies. Allele Pgm1-2 occurred with high frequency in subsp. pyrenaea. It was also but rarely recorded in subsp. balcanica and subsp. olotensis (in one population each). Two loci with five alleles were analysed for LAP (Lap2 and Lap3). The subspecies opicii and balcanica were monomorphic for Lap2-2. This allele had high frequency in subsp. pyrenaea, too, but was less frequent in subsp. amara (Table 3). The zymograms for GDH could be genetically interpreted by two loci $(G d h 1$ and $G d h 2)$ with 6 alleles. In subsp.

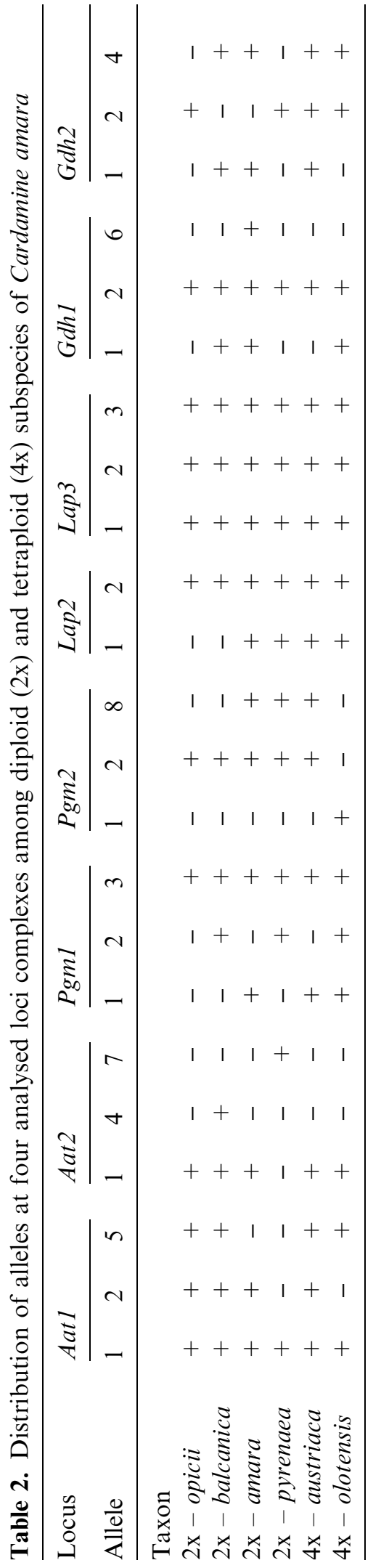


K. Marhold et al.: Isozyme diversity in the Large Bitter-cress

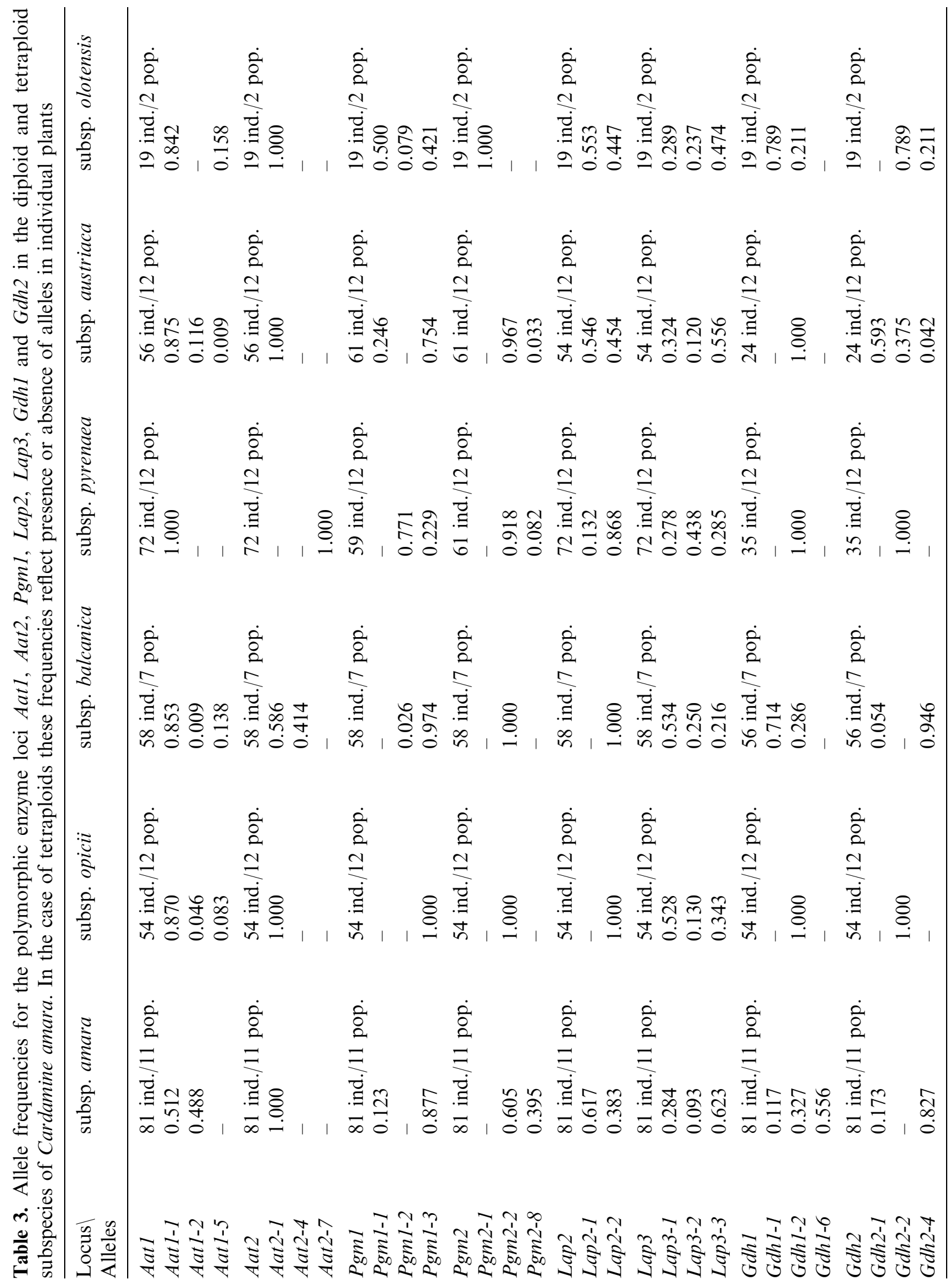


austriaca, Gdh2-1 was nearly fixed, whereas it was detected only in three of the 11 populations surveyed in subsp. amara. These populations were within or close to the range of subsp. austriaca. The allele Gdh2-1 was also found in one population of subsp. balcanica, but neither recorded from subsp. pyrenaea nor from subsp. olotensis (Tables 2 and 3). The allele $G d h 2-2$ was fixed in subsp. opicii and subsp. pyrenaea and was also recorded from subsp. olotensis and subsp. austriaca.

Principal component analyses (PCA). Ordination diagrams based on allelic frequencies (diploid taxa only, not shown) and on presence/absence data (all taxa, Fig. 2) showed similar patterns. C. amara subsp. pyrenaea appeared in a clearly separated position along the first component axis, while subsp. amara, subsp. opicii and subsp. balcanica formed only partly overlapping groupings. The two groups of tetraploids occupied different positions on the ordination diagram. While subsp. olotensis was separated from the rest of material along the third component axis, samples of subsp. austriaca overlapped to a large extent with those of subsp. amara.

Principal coordinate analyses (PCO). PCO of the diploid populations (Fig. 3) based on chord distances of Cavalli-Sforza and Edwards (1967) revealed C. amara subsp. pyrenaea in a clearly separated position along the first axis and subsp. opicii along the first and second axes. $C$. amara subsp. amara and subsp. balcanica partly overlapped, but this overlap was less apparent in the diagram of PCO (not shown) where subsp. pyrenaea was omitted (in order to get a better resolution on the first few axes).

Non-parametric classificatory discriminant analysis. All accessions of C. amara subsp.

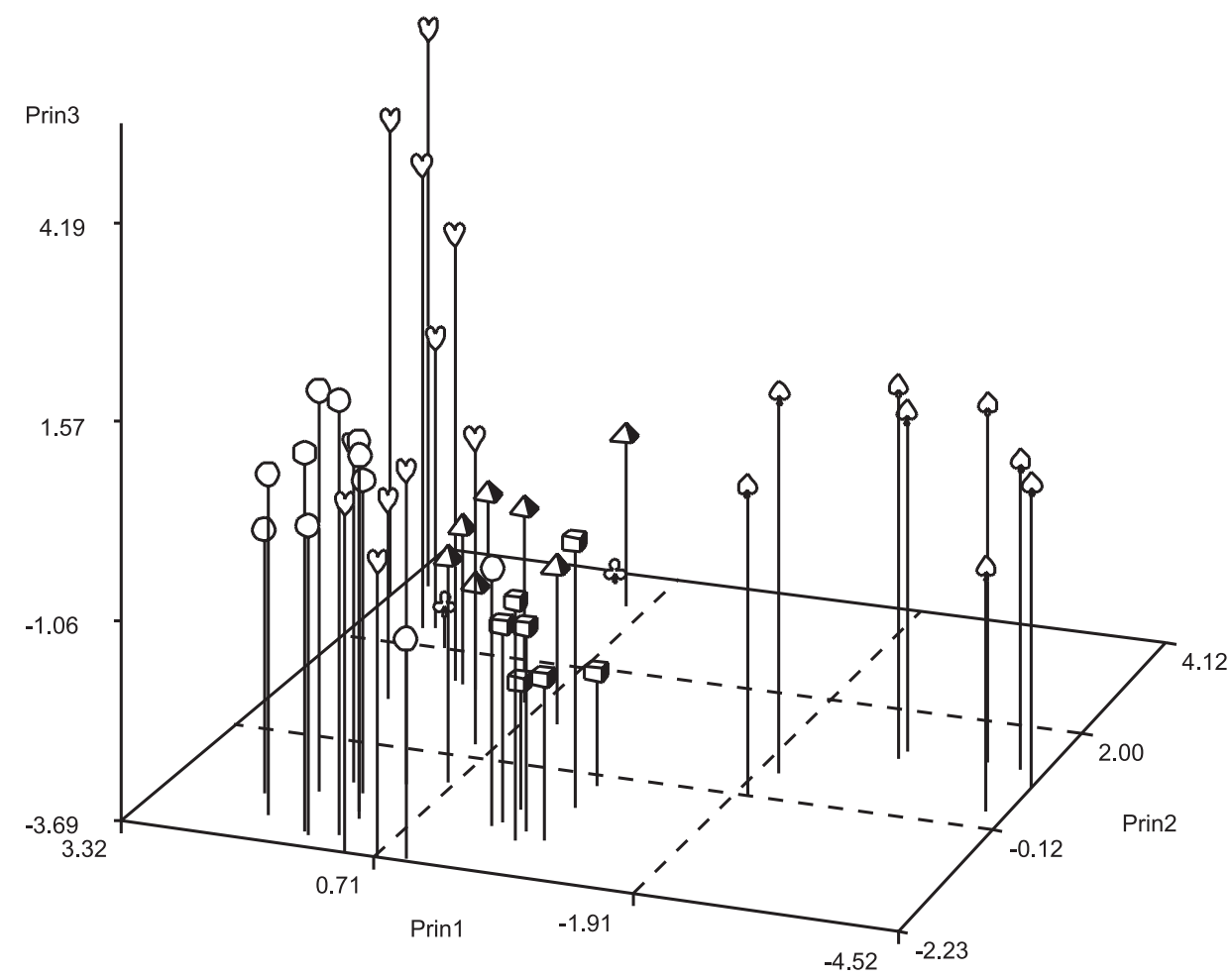

Fig. 2. Principal component analysis of diploid and tetraploid populations of Cardamine amara based on presence/absence of alleles (heart - subsp. amara, pyramid - subsp. balcanica, cube - subsp. opicii, spade subsp. pyrenaea, ball - subsp. austriaca, club - subsp. olotensis). The first three component axes account for $22.2 \%, 13.4 \%$ and $10.6 \%$ of variation respectively 
opicii, subsp. balcanica, subsp. pyrenaea and subsp. olotensis were classified by the classificatory discriminant analysis according to their taxonomic position. Two samples (out of 11) of subsp. amara were incorrectly classified into subsp. austriaca and one sample of subsp. austriaca (out of 12) was incorrectly classified into subsp. opicii.

Cluster analysis. Cluster analysis of the diploid populations (not shown), based on chord distances of Cavalli-Sforza and Edwards (1967) performed by UPGMA clustering method showed only two compact subspecies clusters, namely those composed of subspp. pyrenaea and opicii. Neither populations of subsp. balcanica nor those of subsp. amara formed compact clusters in this analysis. This might be interpreted as a consequence of the wide variation of subsp. amara (corresponding to its large distribution area compared with other subspecies) as well as indicating a close position of subspp. amara and balcanica.

\section{Discussion}

Differentiation at the isozyme level within C. amara reflects to certain extent the morphologically and geographically based subspecies concept. Presence/absence data and differences in allele frequencies characterise most of the infraspecific taxa. There was enough information in the isozyme data (presence/absence of alleles) to discriminate subspecies as was demonstrated by the classificatory discriminant analysis. It should be stressed, however, that this analysis gives more weight to the characters variable among predefined groups (in our case subspecies) than to those with larger withingroup variation. The results of PCA, which gives to all characters equal weight, based on the same data provided more overlapping groupings, but still the tendency of populations for a given subspecies to group together was apparent. The same grouping pattern was apparent also in the analysis of the diploid taxa using

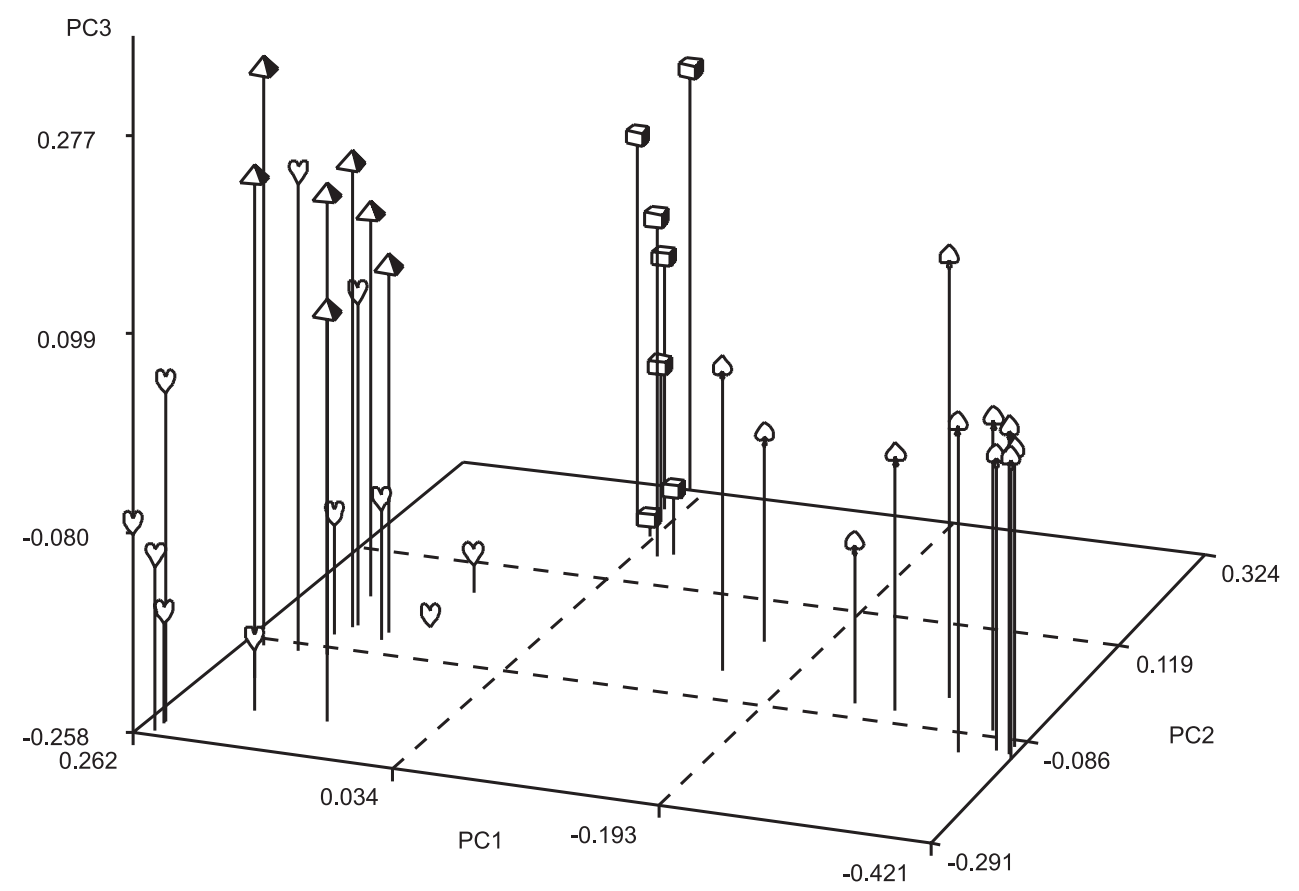

Fig. 3. Principal coordinate analysis of diploid populations of Cardamine amara based on chord distances of Cavalli-Sforza and Edwards (1967) (heart - subsp. amara, pyramid - subsp. balcanica, cube - subsp. opicii, spade - subsp. pyrenaea). The first three coordinate axes account for $21.8 \%, 13.7 \%$ and $8.2 \%$ of variation, respectively 
chord distance and PCO and partly also in the cluster analysis.

From among the diploid taxa, Cardamine amara subsp. pyrenaea was monomorphic for a unique allele (Aat2-7), and subsp. balcanica had a unique allele, too (Aat2-4) which, however, was not fixed in the subspecies. This allele was also detected in C. flexuosa (Hurka, unpubl.). Both subspecies, pyrenaea and balcanica, occur on the margin of the entire distribution range of $C$. amara in areas, which are known as refuge areas during the last glaciation periods (e.g. Hewitt 1999). This coincidence (unique alleles and present distribution area) argues for a relic character and considerable age of these two diploid subspecies. This view is supported by the RAPD data where groupings of subsp. pyrenaea and subsp. balcanica had high bootstrap values (Lihová et al. 2000). The other two diploid subspecies, subsp. amara and subsp. opicii, were not characterised by presence of unique alleles but differed in allele frequencies. The three diploid subspecies $C$. amara subsp. amara, subsp. opicii and subsp. balcanica, although partly differentiated at isozyme loci, still seem to be closely related. Their distribution areas either overlap (subsp. amara vs. subsp. opicii) or they occur in close neighbourhood (subsp. amara vs. subsp. balcanica) and there is probably gene flow between these taxa.

The two tetraploid taxa strongly differ in isozyme pattern which is most probably the result of their different evolutionary histories. C. amara subsp. olotensis is well separated from all other subspecies by the unique allele (Pgm21) and to a certain extent by the frequency of other alleles. On the other hand, tetraploid subsp. austriaca differs only slightly from the diploid subsp. amara. The same pattern was found while evaluating the RAPD data. While subsp. olotensis was clearly different from the rest of the material of the species, subsp. amara and subsp. austriaca did not form separate groupings or clusters in any analysis (Lihová et al. 2000). This is also in accordance with the morphological data and distribution of these taxa. C. amara subsp. olotensis differs from all other subspecies of $C$. amara by the yellow anthers, which is an important and stable character in this genus, as well as in quantitative characters (Lihová et al. 2000). The tetraploid C. amara subsp. austriaca is morphologically very similar to its most likely diploid progenitor, subsp. amara. The only completely reliable character is the size of pollen grains, while other characters, such as the number of leaves, leaflets, and size of some flower parts overlap. Such poor morphological differentiation of this taxon favours a hypothesis of its relatively recent origin. The tetraploid taxa also occupy very different distribution areas. The distribution area of subsp. austriaca coincides with an area heavily affected by the Pleistocene glaciation which lead Marhold (1999) to hypothesise its origin during the last interglacial or in the early postglacial periods. C. amara subsp. olotensis occupies a small relic area in Catalonia, which may indicate its preglacial origin.

The authors would like to thank János Podani for providing the beta version of SYN-TAX 2000 program. Financial support of the Humboldt Foundation, Bonn, Germany enabling K. M. to carry out the research at the University of Osnabrück and the contribution by the Grant Agency VEGA, Bratislava, Slovakia (Grant no. 7080/20) and the Ministry of Education, Youth and Sport of the Czech Republic (Grant no. 1131-4) are greatly acknowledged.

\section{References}

Cavalli-Sforza L. L., Edwards A. W. F. (1967): Phylogenetic analysis: models and estimation procedures. Evolution 21: 550-570.

Franzke A., Hurka H. (2000) Molecular systematics and biogeography of the Cardamine pratensis complex (Brassicaceae). Plant Syst. Evol. 224: 213-234.

Hewitt G. (1999) Post-glacial re-colonization of European biota. Biol. J. Linn. Soc. 68: 7-112.

Hewitt G. (2000) The genetic legacy of the Quaternary ice ages. Nature 405: 907-913.

Klecka W. R. (1980) Discriminant analysis. Sage University Papers, Series: Quantitative applications in the social sciences, no. 19, Beverly Hills and London. 
Koch M., Hurka H. (2000) Isozyme analysis in the polyploid complex Microthlaspi perfoliatum (L.) F. K. Meyer: Morphology, biogeography and evolutionary history. Flora 194: 33-48.

Koch M., Hurka H., Mummenhoff K. (1996) Chloroplast DNA restriction site variation and RAPD-analyses in Cochlearia (Brassicaceae): Biosystematics and speciation. Nord. J. Bot. 16: 595-603.

Lihová J., Marhold K., Neuffer B. (2000) Taxonomy of Cardamine amara (Cruciferae) in the Iberian Peninsula. Taxon 49: 747-763.

Lövkvist B. (1956) The Cardamine pratensis complex. Outlines of its cytogenetics and taxonomy. Symb. Bot. Upsal. 14/2: 1-131.

Marhold K. (1992) A multivariate morphometric study of the Cardamine amara group (Cruciferae) in the Carpathian and Sudeten mountains. Bot. J. Linn. Soc. 110: 121-135.

Marhold K. (1994a) Chromosome numbers of the genus Cardamine L. (Cruciferae) in the Carpathians and Pannonia. Phyton (Horn, Austria) 34: 19-34.

Marhold K. (1994b) Taxonomy of the genus Cardamine L. (Cruciferae) in the Carpathians and Pannonia. I. Cardamine pratensis group. Folia Geobot. Phytotax. 29: 335-374.

Marhold K. (1996) Multivariate morphometric study of the Cardamine pratensis group (Cruciferae) in the Carpathian and Pannonian area. Plant Syst. Evol. 200: 141-159.

Marhold K. (1999) Taxonomic evaluation of the tetraploid populations of Cardamine amara (Brassicaceae) from the Eastern Alps and adjacent areas. Bot. Helv. 109: 67-84.

Marhold K., Ančev M. E. (1999) Cardamine penzesii, a rediscovered taxon of the $C$. pratensis group (Cruciferae). Ann. Bot. Fenn. 36: 171180.

Marhold K., Ančev M. E., Kit Tan (1996) A new subspecies of Cardamine amara (Brassicaceae) from Bulgaria and Greece. Ann. Bot. Fenn. 33: 199-204.
Mummenhoff K., Hurka H. (1995) Allopolyploid origin of Arabidopsis suecica (Fries) Norrlin: Evidence from chloroplast and nuclear genome markers. Bot. Acta 108: 449-456.

Nei M. (1972) Genetic distance between populations. Amer. Naturalist 106: 283-292.

Nei M. (1978) Estimation of average heterozygosity and genetic distance from a small number of individuals. Genetics 89: 583-590.

Podani J. (2001) SYN-TAX 2000, Computer programs for data analysis in ecology and systematics. Scientia Publishing, Budapest.

SAS Institute (1990a) SAS procedures guide, Version 6. Ed. 3. SAS Institute, Cary.

SAS Institute (1990b) SAS/STAT ${ }^{\circledR}$ user's guide, Version 6. Ed. 4. SAS Institute, Cary.

Swofford D. L., Selander R. B. (1989) BIOSYS-1 (Version 1.7). Illinois Natural History Survey, Champaign.

Taberlet P., Fumagalli L., Wust-Saucy A.-G., Cosson J.-F. (1998) Comparative phylogeography and postglacial colonization routes in Europe. Molec. Ecol. 7: 453-464.

Urbanska-Worytkiewicz K., Landolt E. (1974) Biosystematic investigations in Cardamine pratensis L. s.l. I. Diploid taxa from Central Europe and their fertility relationships. Ber. Geobot. Inst. ETH Stiftung Rübel 42: 42-139.

Addresses of the authors: Karol Marhold (e-mail: marhold@savba.sk), Institute of Botany, Slovak Academy of Sciences, Dúbravská cesta 14, SK-842 23 Bratislava, Slovak Republic; Marion Huthmann, Institute of Botany, University of Agricultural Sciences Vienna, Gregor-MendelStrasse 33, A-1080 Wien, Austria; Herbert Hurka, Department of Systematic Botany, University of Osnabrück, Barbarastrasse 11, D-49076 Osnabrück, Germany. 